MATHEMATICS OF COMPUTATION

Volume 69, Number 229, Pages 121-140

S 0025-5718(99)01072-8

Article electronically published on February 19, 1999

\title{
ON THE PROBLEM OF SPURIOUS EIGENVALUES IN THE APPROXIMATION OF LINEAR ELLIPTIC PROBLEMS IN MIXED FORM
}

\author{
DANIELE BOFFI, FRANCO BREZZI, AND LUCIA GASTALDI
}

\begin{abstract}
In the approximation of linear elliptic operators in mixed form, it is well known that the so-called inf-sup and ellipticity in the kernel properties are sufficient (and, in a sense to be made precise, necessary) in order to have good approximation properties and optimal error bounds. One might think, in the spirit of Mercier-Osborn-Rappaz-Raviart and in consideration of the good behavior of commonly used mixed elements (like Raviart-Thomas or Brezzi-Douglas-Marini elements), that these conditions are also sufficient to ensure good convergence properties for eigenvalues. In this paper we show that this is not the case. In particular we present examples of mixed finite element approximations that satisfy the above properties but exhibit spurious eigenvalues. Such bad behavior is proved analytically and demonstrated in numerical experiments. We also present additional assumptions (fulfilled by the commonly used mixed methods already mentioned) which guarantee optimal error bounds for eigenvalue approximations as well.
\end{abstract}

\section{INTRODUCTION}

We consider, as a model problem, the eigenvalue problem for Laplace operator

$$
-\Delta u=\lambda u
$$

in a convex polygonal domain $\Omega$ with suitable boundary conditions (to fix ideas, zero Dirichlet boundary conditions). Here and in the following we will always implicitly assume that eigenvectors (here $u$ ) are looked for among nonzero functions or vectors. We are interested in the approximation of eigenvalue/eigenvector pairs in the so-called mixed formulation that reads:

$$
\begin{aligned}
& \text { find }(\underline{\sigma}, u, \lambda) \text { in } H(\operatorname{div} ; \Omega) \times L^{2}(\Omega) \times \mathbf{R} \text { such that } \\
& \left\{\begin{array}{c}
(\underline{\sigma}, \underline{\tau})+b(\underline{\tau}, u)=0 \quad \forall \underline{\tau} \in H(\operatorname{div} ; \Omega), \\
b(\underline{\sigma}, v)=-\lambda(u, v) \quad \forall v \in L^{2}(\Omega),
\end{array}\right.
\end{aligned}
$$

where, as usual, $(\cdot, \cdot)$ is the inner product in $L^{2}(\Omega)$ or in $L^{2}(\Omega)^{2}$ and $b(\underline{\tau}, v)=$ $(\operatorname{div} \underline{\tau}, v)$. Given finite dimensional subspaces $\Sigma_{h}$ and $V_{h}$ of $H(\operatorname{div} ; \Omega)$ and $L^{2}(\Omega)$

Received by the editor July 8, 1997 and, in revised form, March 17, 1998.

1991 Mathematics Subject Classification. Primary 65N30; Secondary 65N25.

Key words and phrases. Mixed finite element methods, spurious eigenvalues.

Partially supported by I.A.N.-C.N.R. Pavia, by C.N.R. under contracts no. 95.01060.12, 96.03853.CT01, 97.00892.CT01, and by MURST. 
respectively, we consider the approximate problem

$$
\begin{aligned}
& \text { find }\left(\underline{\sigma}_{h}, u_{h}, \lambda_{h}\right) \text { in } \Sigma_{h} \times V_{h} \times \mathbf{R} \text { such that } \\
& \left\{\begin{array}{ccc}
\left(\underline{\sigma}_{h}, \underline{\tau}_{h}\right)+b\left(\underline{\tau}_{h}, u_{h}\right)=0 & \forall \underline{\tau}_{h} \in \Sigma_{h}, \\
b\left(\underline{\sigma}_{h}, v_{h}\right)=-\lambda_{h}\left(u_{h}, v_{h}\right) & \forall v_{h} \in V_{h} .
\end{array}\right.
\end{aligned}
$$

We point out explicitly that the study of the properties of the mixed eigenvalue problem (1.3) enters as a crucial ingredient in the analysis of more complicated applied problems, such as fluid-structure interactions (see e.g. $[2,18,11]$ ) or waveguide resonance (see e.g. $[3,19,5,6]$ ) where, in general, one cannot approximate the problem in the easier and more conventional form (1.1).

We assume that the choice of $\Sigma_{h}$ and $V_{h}$ satisfies the usual stability conditions for mixed discretizations. These are the inf-sup condition:

there exists $\beta>0$, independent of $h$, such that

$$
\inf _{v_{h} \in V_{h}} \sup _{\underline{\tau}_{h} \in \Sigma_{h}} \frac{b\left(\underline{\tau}_{h}, v_{h}\right)}{\left\|\underline{\tau}_{h}\right\|_{H(\operatorname{div} ; \Omega)}\left\|v_{h}\right\|_{L^{2}(\Omega)}} \geq \beta,
$$

and the ellipticity in the kernel:

$$
\begin{aligned}
& \text { there exists } \alpha>0 \text {, independent of } h \text {, such that } \\
& (\underline{\tau}, \underline{\tau}) \geq \alpha\|\underline{\tau}\|_{H(\operatorname{div} ; \Omega)}^{2} \quad \forall \underline{\tau} \in \mathbb{K}_{h},
\end{aligned}
$$

where the discrete kernel $\mathbb{I K}_{h}$ is defined as

$$
I_{h}=\left\{\underline{\tau} \in \Sigma_{h} \text { such that } b(\underline{\tau}, v)=0 \forall v \in V_{h}\right\} .
$$

One might think (in the spirit of [15]: (3.12-16) and Section 7.a) that the above conditions are sufficient in order to give good approximation properties for eigenvalue/eigenvector pairs, whenever $\Sigma_{h}$ and $V_{h}$ are reasonably good approximations of $H(\operatorname{div} ; \Omega)$ and $L^{2}(\Omega)$ respectively. However, this is not the case, as we are going to show in this paper. The reason for failure is hidden in the definition of the compact operator whose spectrum has to be approximated (here the inverse of the Laplace operator) when the mixed formulation is used. To make things clearer, let us introduce the associated boundary value problem in its usual form and in the mixed formulation. Therefore, let $f$ be given in $L^{2}(\Omega)$, and consider the problem

$$
\begin{aligned}
& \text { find } u \in H_{0}^{1}(\Omega) \text { such that } \\
& -\Delta u=f \quad \text { in } \Omega .
\end{aligned}
$$

The unique solution of this problem defines a linear compact operator $T$ from $L^{2}(\Omega)$ into itself: $u=T f$. Consider the same problem in its mixed formulation: now, for a given $f \in L^{2}(\Omega)$, we are looking for a pair $(\underline{\sigma}, u)$ in $H(\operatorname{div} ; \Omega) \times L^{2}(\Omega)$ that satisfies

$$
\begin{cases}(\underline{\sigma}, \underline{\tau})+b(\underline{\tau}, u)=0 & \forall \underline{\tau} \in H(\operatorname{div} ; \Omega), \\ b(\underline{\sigma}, v)=-(f, v) & \forall v \in L^{2}(\Omega) .\end{cases}
$$

Clearly, the $u$ part of the mixed formulation is still given by $u=T f$, while $\underline{\sigma}$ is just the gradient of $u$. However, to be precise, we have now another operator (say $\left.T_{M}\right)$ which is acting from $L^{2}(\Omega)$ into $H(\operatorname{div} ; \Omega) \times L^{2}(\Omega)$. This is not a good setting if we want to look for eigenvalues. Therefore [15], following [14], considers first the product space $H=H(\operatorname{div} ; \Omega) \times L^{2}(\Omega)$ and the operator $T_{H}$ from $H^{\prime}$ into $H$ defined as follows:

$$
\begin{aligned}
& \text { given }(g, f) \text { in } H^{\prime}, \text { find }(\underline{\sigma}, u) \text { in } H \text { such that } \\
& \begin{cases}(\underline{\sigma}, \underline{\tau})+b(\underline{\tau}, u)=\langle g, \underline{\tau}\rangle & \forall \underline{\tau} \in H(\operatorname{div} ; \Omega), \\
b(\underline{\sigma}, v)=-(f, v) & \forall v \in L^{2}(\Omega) .\end{cases}
\end{aligned}
$$


Then they consider the cutoff operator (say, $F$ ) from $L^{2}(\Omega)^{2} \times L^{2}(\Omega)$ into itself, given by $F(g, f)=(0, f)$, and they are led to a generalized eigenvalue problem of the type

$$
(\underline{\sigma}, u)=\lambda T_{H} F(\underline{\sigma}, u)
$$

This is surely correct, but now the operator $T_{H} F$, from $H$ into itself, is not compact any more, and all the subsequent theory of [15] does not apply. We point out that the reason for failure does not originate from an inconvenient way of writing the eigenvalue problem: indeed, the operator $T_{M}$ itself, (mapping $f$ into $(\underline{\sigma}, u)$ ) is not compact as an operator from $L^{2}(\Omega)$ in $H$.

However the results of [15] (Section 7.a) are true (see for instance [1]), since additional properties (besides the inf-sup and the ellipticity in the kernel) hold for their choice of finite element spaces $\Sigma_{h}$ and $V_{h}$, which make the method work. On the other hand, other reasonable choices of $\Sigma_{h}$ and $V_{h}$, although satisfying the inf-sup (1.4) and the ellipticity in the kernel (1.5) properties, fail miserably when applied to eigenvalue problems, as we shall prove analytically and demonstrate by numerical experiments.

An outline of the paper goes as follows. In Section 2 we present an abstract framework in which the problem can be set and we define in a precise way what is to be considered as a good convergence property for eigenvalue/eigenvector pairs.

In Section 3 we recall four types of choices for the spaces $\Sigma_{h}$ and $V_{h}$ : the truly mixed approach, the $Q_{1}-P_{0}$ element on rectangular grids, the $P_{1}-\operatorname{div}\left(P_{1}\right)$ element and the $P_{1}^{*}-Q_{0}$ element on criss-cross grids. We show in particular that the last two elements satisfy both the inf-sup and the ellipticity in the kernel properties. The same is already well known for the truly mixed approach, while the $Q_{1}-P_{0}$ element is only used as an auxiliary step for studying the others, although, being a well-known element, it deserves an analysis for itself: in particular we show that this element, which does not satisfy the usual inf-sup condition for Stokes problem, does indeed satisfy a sort of inf-sup condition in $H(\operatorname{div} ; \Omega)$ that might be of some interest in other applications.

Numerical experiments, reported in Section 4, show however that only the truly mixed approach gives good discrete eigenvalues, while the others exhibit the presence of spurious ones. We stress the fact that the type of failure exhibited by approximations like $P_{1}^{*}-Q_{0}$ or $P_{1}-\operatorname{div}\left(P_{1}\right)$ is, in practice, much more dangerous than the type of failure normally exhibited by choices that do not satisfy the inf-sup condition. Indeed, the latter elements usually have a cloud of spurious eigenvalues that immediately shows the bad quality of the computation. On the other hand, as will become clearer from the numerical experiments shown in Section 4, the former elements have just a few, well-isolated spurious eigenvalues that, when we look at the discrete spectrum, insidiously look like good ones. In Section 5 we prove analytically that the above elements (with the obvious exception of the truly mixed ones) must fail when used to approximate eigenvalues, thus confirming the numerical results of Section 4. Finally, in Section 6, we give some simple sufficient conditions for having good convergence properties of eigenvalue/eigenvector pairs. These sufficient conditions include the usual inf-sup condition and the ellipticity in the kernel plus an additional property regarding the so-called Fortin operator (see [10]). The truly mixed approach satisfies this last property, so that the theory again confirms the numerical results of Section 4. More general sufficient conditions and additional references can be found in [16] and [1]. 


\section{Setting of the Problem}

We are interested in the approximation of the following eigenproblem:

$$
\begin{aligned}
& \text { find }(\underline{\sigma}, u, \lambda) \in H(\operatorname{div} ; \Omega) \times L^{2}(\Omega) \times \mathbf{R} \text { such that } \\
& \left\{\begin{array}{l}
(\underline{\sigma}, \underline{\tau})+(\operatorname{div} \underline{\tau}, u)=0 \quad \forall \underline{\tau} \in H(\operatorname{div} ; \Omega), \\
(\operatorname{div} \underline{\sigma}, v)=-\lambda(u, v) \quad \forall v \in L^{2}(\Omega) .
\end{array}\right.
\end{aligned}
$$

Given finite dimensional subspaces $\Sigma_{h} \subset H(\operatorname{div} ; \Omega)$ and $V_{h} \subset L^{2}(\Omega)$, the discretization of (2.1) is

$$
\begin{aligned}
& \text { find }\left(\underline{\sigma}_{h}, u_{h}, \lambda_{h}\right) \in \Sigma_{h} \times V_{h} \times \mathbf{R} \text { such that } \\
& \left\{\begin{array}{r}
\left(\underline{\sigma}_{h}, \underline{\tau}_{h}\right)+\left(\operatorname{div} \underline{\tau}_{h}, u_{h}\right)=0 \quad \forall \underline{\tau}_{h} \in \Sigma_{h}, \\
\left(\operatorname{div} \underline{\sigma}_{h}, v_{h}\right)=-\lambda_{h}\left(u_{h}, v_{h}\right) \quad \forall v_{h} \in V_{h} .
\end{array}\right.
\end{aligned}
$$

Let $T: L^{2}(\Omega) \rightarrow L^{2}(\Omega)$ be the self-adjoint compact operator defined by

$$
\begin{cases}(\underline{\sigma}, \underline{\tau})+(\operatorname{div} \underline{\tau}, T f)=0 & \forall \underline{\tau} \in H(\operatorname{div} ; \Omega), \\ (\operatorname{div} \underline{\sigma}, v)=-(f, v) & \forall v \in L^{2}(\Omega) .\end{cases}
$$

Then $(\underline{\sigma}, u, \lambda)$ is an eigensolution of problem (2.1) if and only if

$$
\lambda T u=u, \quad \underline{\sigma}=\nabla u .
$$

Hence the eigenvalues $\lambda_{i}(i \in \mathbf{N})$ of problem (2.1) are positive. We denote them by

$$
\begin{aligned}
& 0<\lambda_{1} \leq \lambda_{2} \leq \cdots \leq \lambda_{i} \leq \ldots, \\
& \lim _{i \rightarrow \infty} \lambda_{i}=+\infty
\end{aligned}
$$

For each $i \in \mathbf{N}$ the algebraic multiplicity of $\lambda_{i}$ is one, and $E_{i}$ is the onedimensional eigenspace associated to $\lambda_{i}$. In $L^{2}(\Omega)$ we introduce an orthonormal basis $\left\{u_{i}\right\}$ such that

$$
\begin{aligned}
& E_{i}=\operatorname{span}\left(u_{i}\right), \\
& \left(u_{i}, u_{j}\right)=\delta_{i j} .
\end{aligned}
$$

The following mapping will be useful later on. Let $m: \mathbf{N} \rightarrow \mathbf{N}$ be the application which to every $N$ associates the dimension of the space generated by the eigenspaces of the first $N$ distinct eigenvalues; that is,

$$
\begin{aligned}
& m(1)=\operatorname{dim}\left\{\bigoplus_{i} E_{i}: \lambda_{i}=\lambda_{1}\right\} \\
& m(N+1)=m(N)+\operatorname{dim}\left\{\bigoplus_{i} E_{i}: \lambda_{i}=\lambda_{m(N)+1}\right\} .
\end{aligned}
$$

Clearly, $\lambda_{m(1)}, \ldots, \lambda_{m(N)}(N \in \mathbf{N})$ will now be the first $N$ distinct eigenvalues of $(2.1)$.

Let us denote by $T_{h}: L^{2}(\Omega) \rightarrow L^{2}(\Omega)$ the discrete counterpart of $T$, defined by

$$
\begin{cases}\left(\underline{\sigma}_{h}, \underline{\tau}_{h}\right)+\left(\operatorname{div} \underline{\tau}_{h}, T_{h} f\right)=0 & \forall \underline{\tau}_{h} \in \Sigma_{h}, \\ \left(\operatorname{div} \underline{\sigma}_{h}, v_{h}\right)=-\left(f, v_{h}\right) & \forall v_{h} \in V_{h} .\end{cases}
$$

Then $\left\{T_{h}\right\}$ is a family of self-adjoint compact operators with finite-dimensional range in $L^{2}(\Omega)$. As in the continuous case, $\left(\underline{\sigma}_{h}, u_{h}, \lambda_{h}\right) \in \Sigma_{h} \times V_{h} \times \mathbf{R}$ is an eigensolution of problem (2.2) if and only if

$$
\lambda_{h} T_{h} u_{h}=u_{h}, \quad \underline{\sigma}_{h}=\nabla_{h} u_{h},
$$

with $\nabla_{h}$ a suitable discretization of $\nabla$.

Let $\operatorname{dim} V_{h}=N(h)$; then $T_{h}$ admits $N(h)$ real positive eigenvalues

$$
\lambda_{1}^{h} \leq \cdots \leq \lambda_{i}^{h} \leq \cdots \leq \lambda_{N(h)}^{h} .
$$


The associated discrete eigenfuntions $u_{i}^{h}, i=1, \ldots, N(h)$, give rise to an orthonormal basis in $V_{h}$ with respect to the scalar product of $L^{2}(\Omega)$. Let $E_{i}^{h}=$ $\operatorname{span}\left(u_{i}^{h}\right)$ denote the discrete eigenspace associated to $\lambda_{i}^{h}$.

A classical assumption in the theory of spectrum perturbation is the uniform convergence of the operators, that is,

$$
\lim _{h \rightarrow 0}\left\|T-T_{h}\right\|_{\mathcal{L}\left(L^{2}(\Omega)\right)}=0 .
$$

As a consequence of (2.11), we have

$$
\begin{aligned}
& \forall \varepsilon>0, \forall N \in \mathbf{N} \quad \exists h_{0}>0 \text { such that } \forall h \leq h_{0} \\
& \quad \max _{i=1, \ldots, m(N)}\left|\lambda_{i}-\lambda_{i}^{h}\right| \leq \varepsilon, \\
& \hat{\delta}\left(\bigoplus_{i=1}^{m(N)} E_{i}, \bigoplus_{i=1}^{m(N)} E_{i}^{h}\right) \leq \varepsilon,
\end{aligned}
$$

where $\hat{\delta}(E, F)$, for $E$ and $F$ linear subspaces of $L^{2}(\Omega)$, represents the gap between $E$ and $F$ and is defined by

$$
\begin{aligned}
& \hat{\delta}(E, F)=\max [\delta(E, F), \delta(F, E)], \\
& \delta(E, F)=\sup _{u \in E,\|u\|=1} \inf _{v \in F}\|u-v\|_{0} .
\end{aligned}
$$

In (2.13) $\|\cdot\|_{0}$ stands for the $L^{2}$-norm.

We conclude with an additional notation that will be constantly used in the following. Although the definition of the space $V_{h} \subset L^{2}(\Omega)$ will change from one example of finite element approximation to the next, we shall always denote by the symbol $\mathbb{P}_{h}$ the $L^{2}(\Omega)$-projection onto $V_{h}$, that is,

$$
\int_{\Omega}\left(v-\mathbb{P}_{h} v\right) v_{h} d x=0 \quad \forall v \in L^{2}(\Omega), \forall v_{h} \in V_{h}
$$

\section{Various ChOices of SPACES}

In this section we present several possible choices for the spaces $\Sigma_{h} \subset H(\operatorname{div} ; \Omega)$ and $V_{h} \subset L^{2}(\Omega)$. For each choice of spaces, we test the validity of the following two hypotheses:

$$
\begin{aligned}
& \text { there exists } \alpha>0 \text {, independent of } h, \text { such that } \\
& (\underline{\tau}, \underline{\tau}) \geq \alpha\|\underline{\tau}\|_{\text {div }}^{2} \quad \forall \underline{\tau} \in \mathbb{I} K_{h},
\end{aligned}
$$

where the discrete kernel $\mathbb{I}_{h}$ is defined as

$$
I_{h}=\left\{\underline{\tau} \in \Sigma_{h} \text { such that }(\operatorname{div} \underline{\tau}, v)=0 \forall v \in V_{h}\right\},
$$

and

$$
\begin{aligned}
& \text { there exists } \beta>0, \text { independent of } h \text {, such that } \\
& \inf _{v_{h} \in V_{h}} \sup _{\underline{\tau}_{h} \in \Sigma_{h}} \frac{\operatorname{div}\left(\underline{\tau}_{h}, v_{h}\right)}{\left\|\underline{\tau}_{h}\right\|_{\operatorname{div}}\left\|v_{h}\right\|_{0}} \geq \beta .
\end{aligned}
$$

In (3.1) and (3.2), $\|\cdot\|_{\text {div }}$ denotes the graph norm in $H(\operatorname{div} ; \Omega)$.

It is well known that the assumptions (3.1) and (3.2) ensure the existence, uniqueness and stability of the solution of (2.8) (see [8]). We shall see that these hypotheses are not sufficient in order to have a good mixed approximation of the spectrum for the Laplace operator. 
3.1. The mixed approach. Let us consider classical approximations of $H(\operatorname{div} ; \Omega)$, among which there are, for instance, the elements introduced by Raviart-Thomas (RT), Brezzi-Douglas-Marini (BDM) and Brezzi-Douglas-Fortin-Marini (BDFM). For a unified presentation we refer to [8]. In this subsection $\Sigma_{h}$ will be one of the mixed finite element spaces mentioned above. Correspondingly $V_{h}$ will be the space $\operatorname{div} \Sigma_{h}$, which contains piecewise polynomials of a certain degree $k$.

We recall the main properties which are enjoied by the pair $\left(\Sigma_{h}, V_{h}\right)$ and which turn out to be crucial for the eigenvalue approximation.

The first property concerns the so-called Fortin's operator $\Pi_{h}$, acting from $W:=$ $H(\operatorname{div} ; \Omega) \cap\left(L^{s}(\Omega)\right)^{2}\left(s>2\right.$ fixed) into $\Sigma_{h}$. This operator, defined using suitable degrees of freedom, gives us the commuting diagram



This implies that (3.1) and (3.2) are satisfied (see e.g. [8], p. 131).

The following approximation property holds for $1 \leq m \leq k+1$ :

$$
\left\|\underline{\tau}-\Pi_{h} \underline{\tau}\right\|_{0} \leq C h^{m}|\underline{\tau}|_{m} .
$$

Let $f \in L^{2}(\Omega)$. Then, due to the regularity assumptions on $\Omega, T f$ belongs to $H^{2}(\Omega)$. Hence we have (see [8], (IV.1.31))

$$
\left\|T f-T_{h} f\right\|_{0} \leq C h\left(\|\underline{\nabla} f f\|_{1}+\|T f\|_{1}\right) .
$$

This last equation means that $T_{h}$ converges uniformly to $T$, see (2.11).

3.2. The $Q_{1}-P_{0}$ element on a rectangular mesh. Let us consider a square domain $\Omega$ and a partition of $\Omega$ into $N \times N$ macroelements, each made of $2 \times 2$ squares. As usual $K$ will denote an element (of length $h$ ) of the triangulation $\mathcal{T}_{h}$.

We consider the following approximating spaces:

$$
\begin{aligned}
& \Sigma_{Q}=\left\{\underline{\tau}_{h} \in\left[C^{0}(\Omega)\right]^{2}:\left.\underline{\tau}_{h}\right|_{K} \in\left[Q_{1}(K)\right]^{2} \forall K \in \mathcal{T}_{h}\right\}, \\
& V_{h}=\left\{v_{h}:\left.v_{h}\right|_{K} \in P_{0}(K) \forall K \in \mathcal{T}_{h}\right\} .
\end{aligned}
$$

This choice of spaces does not satisfy the inf-sup condition (3.2). However, we prove a modified inf-sup condition involving a mesh dependent norm. This result will be useful in order to analyze the element of the next subsection.

A local basis of $V_{h}$ on a macroelement is shown in Figure 1. Notice that the basis we have chosen is orthogonal.

Let $V_{J P}$ be the subspace of $V_{h}$ locally generated by $v_{i}, i=1,2,3$. In the paper by Johnson and Pitkäranta [13], it has been proved that the spaces $\Sigma_{Q}$ and $V_{J P}$ satisfy the inf-sup condition as follows.

Lemma 3.1. There exists a constant $C$ independent of $h$ such that

$$
\sup _{\underline{\tau}_{h} \in \Sigma_{Q}} \frac{\left(\operatorname{div} \underline{\tau}_{h}, v_{h}\right)}{\left\|\underline{\tau}_{h}\right\|_{1}} \geq C\left\|v_{h}\right\|_{0}
$$

for all $v_{h} \in V_{J P}$. 

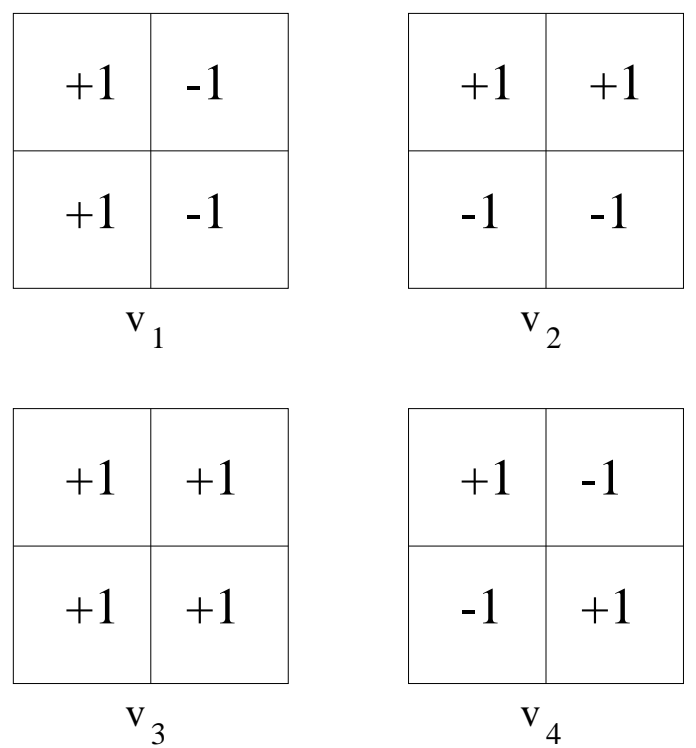

Figure 1. Basis for $V_{h}$ on a macroelement of $2 \times 2$ squares

In Lemma 3.1 the space $V_{J P}$ cannot be replaced by $V_{h}$. However, if the norm in $H^{1}(\Omega)$ is replaced by a mesh dependent one, then it is possible to verify the inf-sup condition. We set

$$
\left\|\underline{\tau}_{h}\right\|_{h}=\left(\left\|\underline{\tau}_{h}\right\|_{0}^{2}+\left\|\mathbb{P}_{h} \operatorname{div} \underline{\tau}_{h}\right\|_{0}^{2}\right)^{1 / 2}
$$

then the following theorem holds true.

Theorem 3.2. There exists a constant $C$ independent of $h$ such that for every $v_{h} \in V_{h}$ there exists $\underline{\tau}_{h} \in \Sigma_{Q}$ verifying

$$
\left(\operatorname{div} \underline{\tau}_{h}, v_{h}\right) \geq\left\|v_{h}\right\|_{0}^{2}, \quad\left\|\underline{\tau}_{h}\right\|_{h} \leq C\left\|v_{h}\right\|_{0} .
$$

Proof. We work on macroelements of $2 \times 2$ squares. Let us split a given $v_{h} \in V_{h}$ into the sum of $v_{h}^{b} \in V_{J P}$ and $v_{h}^{c}$ which is locally generated by $v_{4}$ (see Figure 1), so that $v_{h}=v_{h}^{c}+v_{h}^{b}$.

Using Lemma 3.1, there exists $\underline{\tau}_{h}^{b} \in \Sigma_{Q}$ such that

$$
\left(\operatorname{div} \underline{\tau}_{h}^{b}, v_{h}^{b}\right) \geq\left\|v_{h}^{b}\right\|_{0}^{2}, \quad\left\|\underline{\tau}_{h}^{b}\right\|_{1} \leq C_{1}\left\|v_{h}^{b}\right\|_{0} .
$$

The main step of the proof is to construct an element $\underline{\tau}_{h}^{c} \in \Sigma_{Q}$ such that

$$
\mathbb{P}_{h} \operatorname{div} \underline{\tau}_{h}^{c}=v_{h}^{c}, \quad\left\|\underline{\tau}_{h}^{c}\right\|_{0} \leq C_{2}\left\|v_{h}^{c}\right\|_{0} .
$$

We fix our attention on the row of macroelements lying in the strip $S_{j}=$ ] $0,2 N h[\times] 2(j-1) h, 2 j h\left[\right.$. On each macroelement, $v_{h}^{c}$ is equal to $v_{4}$ multiplied by a certain constant. We denote by $c_{i}$ the value of this constant on the $i$ th macroelement, $i=1, \ldots, N$. In the row we have considered, we define $\underline{\tau}_{h}^{c}$ using the $2 N$ degrees of freedom drawn in Figure 2. At all other nodes it is set equal to zero. Since $v_{h}^{c}$ is piecewise constant, an explicit computation shows that $\underline{\tau}_{h}^{c}$ can be defined 


\begin{tabular}{|l|l|l|l|l|l|l|}
\hline $\mathrm{c}_{1}$ & $-\mathrm{c}_{1}$ & $\mathrm{c}_{2}$ & $-\mathrm{c}_{2}$ & & & \\
\hline$-\mathrm{c}_{1}$ & $\mathrm{c}_{1}$ & $-\mathrm{c}_{2}$ & $\mathrm{c}_{2}$ & & $-\mathrm{c}_{\mathrm{N}}$ \\
\hline
\end{tabular}

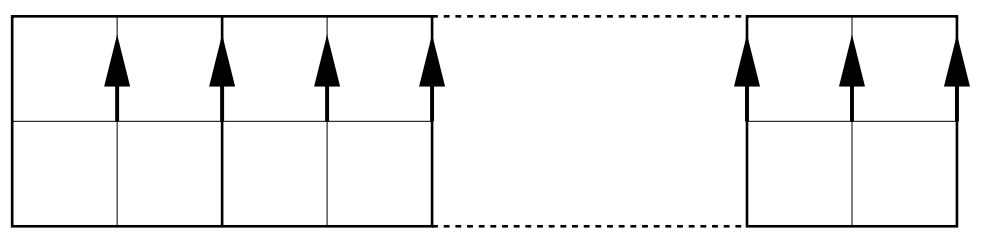

FiguRE 2. Degrees of freedom for a row of checkerboards

as follows, in order to have $\left.\left(\mathbb{P}_{h} \operatorname{div} \underline{\tau}_{h}^{c}\right)\right|_{K}=\left.\left(v_{h}^{c}\right)\right|_{K}$ for each square $K \subset S_{j}$ :

$$
\begin{aligned}
& \underline{\tau}_{h}^{c}((2 i-1) h,(2 j-1) h)=2 h\left(0,-c_{i}-2 \sum_{\ell=1}^{i-1} c_{\ell}\right), \\
& \underline{\tau}_{h}^{c}(2 i h,(2 j-1) h)=2 h\left(0,2 \sum_{\ell=1}^{i} c_{\ell}\right) .
\end{aligned}
$$

The $L^{2}(\Omega)$-norm in $S_{j}$ of $v_{h}^{c}$ and $\underline{\tau}_{h}^{c}$ can be evaluated as follows:

$$
\begin{aligned}
\left\|v_{h}^{c}\right\|_{0, S_{j}}^{2} & =4 h^{2} \sum_{i=1}^{N} c_{i}^{2} \\
\left\|\underline{\tau}_{h}^{c}\right\|_{0, S_{j}}^{2} & \leq C h^{4} \sum_{i=1}^{N}\left(\sum_{\ell=1}^{i} c_{\ell}\right)^{2} \\
& \leq C h^{4} \sum_{i=1}^{N}\left(\sum_{\ell=1}^{i} 1\right)\left(\sum_{\ell=1}^{i} c_{\ell}^{2}\right) \leq C h^{2} \sum_{i=1}^{N} c_{i}^{2} .
\end{aligned}
$$

Then we have

$$
\left\|\underline{\tau}_{h}^{c}\right\|_{0, S_{j}}^{2} \leq C\left\|v_{h}^{c}\right\|_{0, S_{j}}^{2}
$$

which implies the corresponding bound in the whole domain (3.11).

We are now in position to conclude the proof. Let $\gamma=\left(1+C_{1}^{2}\right) / 2$, where $C_{1}$ is defined in (3.10). Taking $\underline{\tau}_{h}=\gamma \underline{\tau}_{h}^{c}+\underline{\tau}_{h}^{b}$ and noting that $\left(\operatorname{div} \underline{\tau}_{h}^{c}, v_{h}^{b}\right)=\left(v_{h}^{c}, v_{h}^{b}\right)=0$, we obtain

$$
\begin{aligned}
& \left(\operatorname{div} \underline{\tau}_{h}, v_{h}\right)=\gamma\left(\operatorname{div} \underline{\tau}_{h}^{c}, v_{h}^{c}\right)+\left(\operatorname{div} \underline{\tau}_{h}^{b}, v_{h}^{b}\right)+\left(\operatorname{div} \underline{\tau}_{h}^{b}, v_{h}^{c}\right) \\
& \quad \geq \gamma\left\|v_{h}^{c}\right\|_{0}^{2}+\left\|v_{h}^{b}\right\|_{0}^{2}-\left\|\operatorname{div} \underline{\tau}_{h}^{b}\right\|_{0}\left\|v_{h}^{c}\right\|_{0} \geq \gamma\left\|v_{h}^{c}\right\|_{0}^{2}+\left\|v_{h}^{b}\right\|_{0}^{2}-C_{1}\left\|v_{h}^{b}\right\|_{0}\left\|v_{h}^{c}\right\|_{0} \\
& \quad \geq\left(\gamma-\frac{C_{1}^{2}}{2}\right)\left\|v_{h}^{c}\right\|_{0}^{2}+\frac{1}{2}\left\|v_{h}^{b}\right\|_{0}^{2} \geq \frac{1}{2}\left\|v_{h}\right\|_{0}^{2}, \\
& \left\|\underline{\tau}_{h}\right\|_{h}^{2} \leq \gamma\left\|\underline{\tau}_{h}^{c}\right\|_{0}^{2}+\gamma\left\|\mathbb{P}_{h} \operatorname{div} \underline{\tau}_{h}^{c}\right\|_{0}^{2}+\left\|\underline{\tau}_{h}^{b}\right\|_{1}^{2} \leq C\left(\left\|v_{h}^{c}\right\|_{0}^{2}+\left\|v_{h}^{b}\right\|_{0}^{2}\right)=C\left\|v_{h}\right\|_{0}^{2} .
\end{aligned}
$$




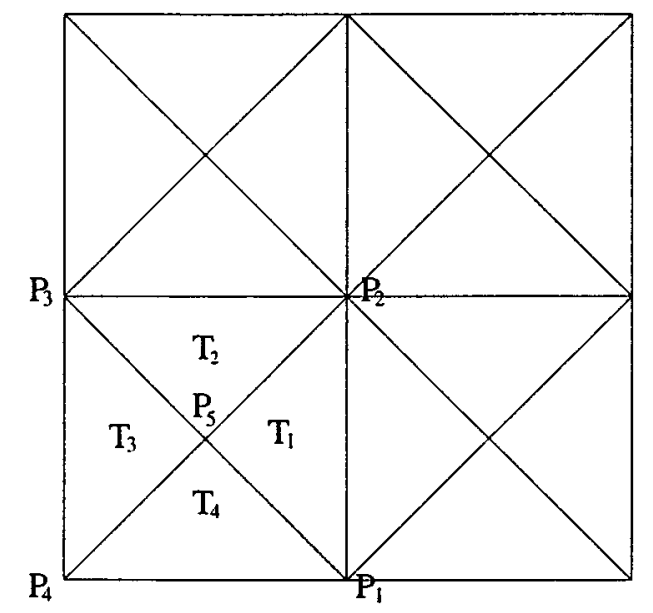

Figure 3. A criss-cross macroelement

Remark 3.3. Theorem 3.2 does not imply the inf-sup condition (3.2), since in (3.9) the mesh dependent norm $\|\cdot\|_{h}$ is used instead of the norm of $H(\operatorname{div} ; \Omega)$. Theorem 3.2 is however crucial for the analysis of the element presented in the next subsection.

3.3. The $P_{1}-\operatorname{div}\left(P_{1}\right)$ element on a criss-cross mesh. It is well known that the $P_{1}-P_{0}$ element does not satisfy the inf-sup condition (3.2) on a criss-cross mesh (see, for instance, [8]). Indeed there exists a piecewise constant function which is orthogonal to the divergence of every continuous piecewise linear vector fields. Hence we define $V_{h}$ to be the space of the divergences of all continuous piecewise linear vector fields.

For this element we are able to prove both the ellipticity in the kernel (3.1) and the inf-sup (3.2) conditions.

Let us consider a square domain $\Omega$, which is split into $2 N \times 2 N$ squares; each of them is then partitioned into four triangles by its diagonals (see Figure 3). We denote by $Q \in \mathcal{Q}_{h}$ the squares and by $T \in \mathcal{T}_{h}$ the triangles.

We introduce the following finite element spaces:

$$
\begin{aligned}
& \Sigma_{h}=\left\{\underline{\tau}_{h} \in\left[C^{0}(\Omega)\right]^{2}:\left.\underline{\tau}_{h}\right|_{T} \in\left[P_{1}(T)\right]^{2} \forall T \in \mathcal{T}_{h}\right\}, \\
& V_{h}=\operatorname{div}\left(\Sigma_{h}\right) .
\end{aligned}
$$

In the following theorem we observe that our choice (3.13) satisfies (3.1).

Theorem 3.4. The spaces $\Sigma_{h}$ and $V_{h}$ defined in (3.13) satisfy the ellipticity in the kernel property (3.1).

Proof. The discrete kernel $\mathbb{I}_{h}$, due to the definition of $V_{h}$, is contained in the continuous kernel.

In the following lemma, we characterize the space $V_{h}$.

Lemma 3.5. The elements of $V_{h}$ are piecewise constants and are characterized by the following relation between the values on each triangle in a criss-cross square (see Figure 4):

$$
a+c=b+d .
$$




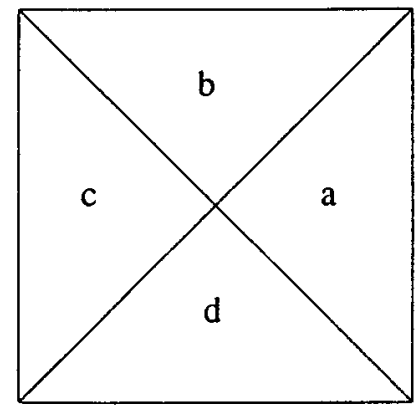

Figure 4. Piecewise constants on a criss-cross square
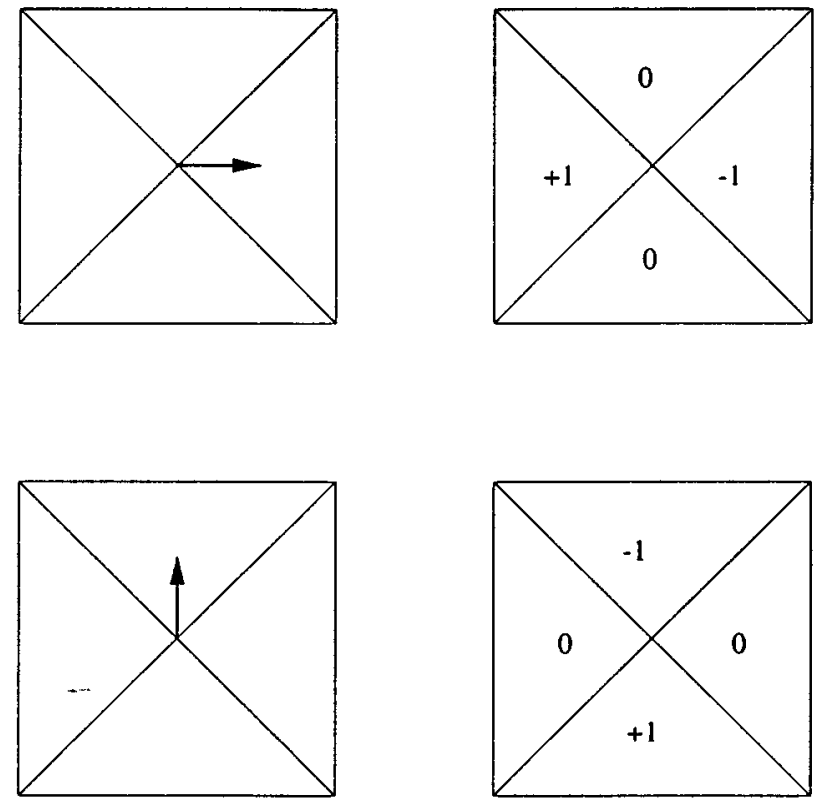

Figure 5. The divergence of $B$

Proof. In Figures 5 and 6 the divergence of some basis function in $\Sigma_{h}$ are represented. By linearity the result follows immediately for the divergence of every vector in $\Sigma_{h}$.

We set

$$
\begin{aligned}
V_{c} & =\left\{v \in V_{h}:\left.v\right|_{Q} \text { is constant } \forall Q \in \mathcal{Q}_{h}\right\}, \\
V_{b} & =\left\{v \in V_{h}: \int_{Q} v=0 \forall Q \in \mathcal{Q}_{h}\right\} .
\end{aligned}
$$

It is immediate to see that $V_{b}$, in each square $Q$, has dimension two, a basis being given by the two modes on the right-hand side of Figure 5. 

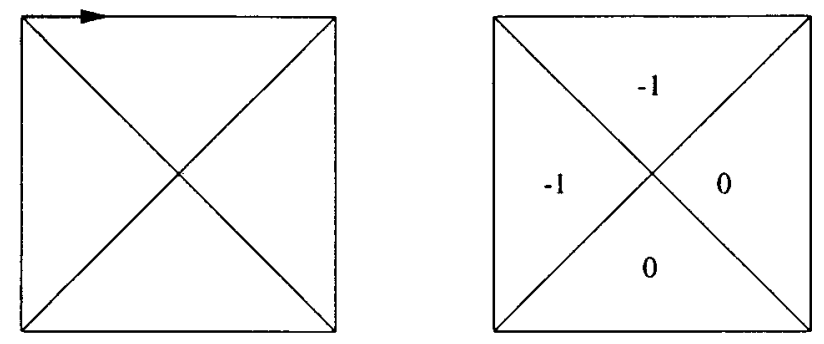

Figure 6 . The divergence of an element in $\Sigma_{h}$

Lemma 3.6. The following orthogonal decomposition holds true:

$$
V_{h}=V_{c} \oplus V_{b}, \quad \text { with } V_{c} \perp V_{b} .
$$

Proof. Given $v \in V_{h}$, let us consider the element $v_{c} \in V_{c}$ such that

$$
\int_{Q} v_{c}=\int_{Q} v \quad \forall Q \in \mathcal{Q}_{h} .
$$

Then $v_{b}=v-v_{c}$ is an element of $V_{b}$. It is obvious that with this construction the decomposition is unique. Moreover,

$$
\int_{\Omega} v_{c} v_{b}=0 .
$$

We set

$$
B=\operatorname{span}\left\{\underline{\tau} \in \Sigma_{h}: \operatorname{supp} \underline{\tau} \subseteq Q, Q \in \mathcal{Q}_{h}\right\} .
$$

The divergence of a local basis in $B$ is represented in Figure 5. The inclusion $\operatorname{div} B \subseteq V_{b}$ is obvious.

The following lemma is also obvious from Figure 5 and a simple scaling argument.

Lemma 3.7. The divergence operator is injective and surjective between $B$ and $V_{b}$. That is, for each $v_{b} \in V_{b}$ there exists a unique $\underline{b} \in B$ which satisfies

$$
\operatorname{div} \underline{b}=v_{b} .
$$

Moreover, there exists $C$ independent of $h$ such that

$$
\|\underline{b}\|_{0} \leq C h\left\|v_{b}\right\|_{0} .
$$

We set

$$
\Sigma_{c}=\left\{\underline{\tau} \in \Sigma_{h}: \operatorname{div} \underline{\tau} \in V_{c}\right\} .
$$

Lemma 3.8. The following decomposition holds true:

$$
\Sigma_{h}=\Sigma_{c} \oplus B .
$$

Moreover, the following orthogonalities are satisfied:

$$
\begin{array}{ll}
\left(\operatorname{div} \underline{\tau}_{c}, v_{b}\right)=0 & \forall \underline{\tau} \underline{\tau}_{c} \in \Sigma_{c}, \forall v_{b} \in V_{b}, \\
\left(\operatorname{div} \underline{b}, v_{c}\right)=0 & \forall \underline{b} \in B, \forall v_{c} \in V_{c} .
\end{array}
$$


Proof. Let us consider $\underline{\tau} \in \Sigma_{h}$. By definition of $V_{h}$ and Lemma 3.6 there exist $v_{c} \in V_{c}$ and $v_{b} \in V_{b}$ such that $\operatorname{div} \underline{\tau}=v_{c}+v_{b}$. Let $\underline{b}$ be the unique element of $B$ such that $\operatorname{div} \underline{b}=v_{b}$ (see Lemma 3.7). We define $\underline{\tau}_{c}=\underline{\tau}-\underline{b}$. Then $\operatorname{div} \underline{\tau}_{c}=v_{c}$, and hence $\underline{\tau}_{c} \in \Sigma_{c}$. The decomposition is unique by construction. Finally, the orthogonalities (3.25) are straightforward.

The following lemma will be useful in order to apply the results of the previous subsection.

Lemma 3.9. Let $\Sigma_{Q}$ be the space defined in (3.6) (that is, locally $Q_{1}$ ). Then for each $\underline{\xi}_{h} \in \Sigma_{Q}$ there exists $\underline{\tau}_{h} \in \Sigma_{c}$ satisfying

$$
\begin{aligned}
& \left(\operatorname{div} \underline{\tau}_{h}, v\right)=\left(\operatorname{div} \underline{\xi}_{h}, v\right) \quad \forall v \in V_{c}, \\
& \left\|\underline{\tau}_{h}\right\|_{r} \leq C\left\|\underline{\xi}_{h}\right\|_{r} \quad(r=0,1),
\end{aligned}
$$

with $C$ independent of $h$. Moreover, $\underline{\tau}_{h}$ can be chosen so that it attains the same values as $\underline{\xi}_{h}$ at all nodes of $\mathcal{Q}_{h}$.

Proof. For a $\underline{\tau}_{h} \in \Sigma_{c}$ equation (3.26) means that $\operatorname{div} \underline{\tau}_{h}$ is the $L^{2}(\Omega)$-projection of $\operatorname{div} \xi_{h}$ onto $V_{c}$. Let $Q$ be a square in $\mathcal{Q}_{h}$ (see Figure 3). Let us denote by $\varphi_{i}$, $i=1, \ldots, 4$, the piecewise linear basis functions associated to the vertices $P_{1}, P_{2}$, $P_{3}$ and $P_{4}$ of $Q$ and by $\varphi_{5}$ the one associated to the center. On $Q, \underline{\tau}_{h} \in \Sigma_{h}$ can be represented as follows:

$$
\underline{\tau}_{h}=\sum_{i=1}^{5}\left(u_{i}, v_{i}\right) \varphi_{i} .
$$

We take

$$
\left(u_{i}, v_{i}\right)=\underline{\xi}_{h}\left(P_{i}\right), \quad i=1, \ldots, 4 .
$$

Whatever the value of $\left(u_{5}, v_{5}\right)$ may be, the mean value of $\operatorname{div} \underline{\tau}_{h}$ on $Q$ is equal to the mean value of $\operatorname{div} \underline{\xi}_{h}$, thanks to the Gauss theorem. Hence condition (3.26) is satisfied. We have only to fix the value of $\underline{\tau}_{h}$ in $P_{5}$ in order to achieve that it belongs to $\Sigma_{c}$. A straightforward calculation leads to

$$
\begin{aligned}
& u_{5}=\left(u_{1}-v_{1}+u_{2}+v_{2}+u_{3}-v_{3}+u_{4}+v_{4}\right) / 4, \\
& v_{5}=\left(-u_{1}+v_{1}+u_{2}+v_{2}-u_{3}+v_{3}+u_{4}+v_{4}\right) / 4 .
\end{aligned}
$$

A scaling argument gives the bounds (3.27).

We state the main result of this section.

Theorem 3.10. The following inf-sup condition holds true:

$$
\inf _{v \in V_{h}} \sup _{\underline{\tau} \in \Sigma_{h}} \frac{(\operatorname{div} \underline{\tau}, v)}{\|\underline{\tau}\|_{\operatorname{div}}\|v\|_{0}} \geq \beta_{0}>0, \quad \forall h>0 .
$$

Proof. Given $v_{h} \in V_{h}$, let $v_{c} \in V_{c}$ and $v_{b} \in V_{b}$ be such that $v_{h}=v_{c}+v_{b}$. Theorem 3.2 and Lemma 3.7 imply the existence of $\underline{\xi}_{h} \in \Sigma_{Q}$ and $\underline{b} \in B$, respectively, satisfying

$$
\begin{array}{ll}
\left(\operatorname{div} \underline{\xi}_{h}, v_{c}\right) \geq\left\|v_{c}\right\|_{0}^{2}, & \left(\operatorname{div} \underline{b}, v_{b}\right)=\left\|v_{b}\right\|_{0}^{2}, \\
\left\|\underline{\xi}_{h}\right\|\left\|_{\text {div }} \leq C_{1}\right\| v_{c} \|_{0}, & \|\underline{b}\|_{\text {div }} \leq C_{2} h\left\|v_{b}\right\|_{0} .
\end{array}
$$


Using Lemma 3.9, there exists $\underline{\tau}_{c} \in \Sigma_{c}$ such that

$$
\begin{aligned}
& \left(\operatorname{div} \underline{\tau}_{c}, v_{c}\right)=\left(\operatorname{div} \underline{\xi}_{h}, v_{c}\right), \\
& \left\|\underline{\tau}_{c}\right\|_{H(\operatorname{div} ; \Omega)} \leq C\left\|\underline{\xi}_{h}\right\|_{H(\operatorname{div} ; \Omega)} .
\end{aligned}
$$

It is not difficult to verify that, defining $\underline{\tau}_{h}=\underline{\tau}_{c}+\underline{b}$, thanks to Lemma 3.8, one has

$$
\left(\operatorname{div} \underline{\tau}_{h}, v_{h}\right) \geq\left\|v_{h}\right\|_{0}^{2}, \quad\left\|\underline{\tau}_{h}\right\|_{\text {div }} \leq C\left\|v_{h}\right\|_{0}
$$

Remark 3.11. Inequality (3.31) is optimal; in fact it cannot be improved, since for each $h$ there exists $\tilde{v}_{h} \in V_{h}$ such that

$$
\left(\operatorname{div} \underline{\tau}_{h}, \tilde{v}_{h}\right) \leq C\left\|\underline{\tau}_{h}\right\|_{0}\left\|\tilde{v}_{h}\right\|_{0} \quad \forall \underline{\tau}_{h} \in \Sigma_{h} .
$$

This inequality was proved by Qin in his Ph.D. dissertation [17], using an idea of Boland and Nicolaides [7] (see also [12]). In particular, the element $\tilde{v}_{h}$ is a properly chosen linear combination of checkerboards on the macroelements.

Remark 3.12. In the proof of Theorem 3.2, the normal component of vectors in $\Sigma_{h}$ has not been used, while we used the tangential component on the boundary (see Figure 2). Actually the proof could be completed without using any boundary degrees of freedom for the space $\Sigma_{h}$. It turns out that the spaces $\Sigma_{h} \cap H_{0}^{1}(\Omega)^{2}$ and $\operatorname{div}\left(\Sigma_{h} \cap H_{0}^{1}(\Omega)^{2}\right)$ satisfy the inf-sup condition (3.2).

3.4. The $P_{1}^{*}-Q_{0}$ element on a criss-cross mesh. Let us consider again the $P_{1}-\operatorname{div}\left(P_{1}\right)$ element of the previous section. During the analysis of this element, we introduced the subspace $\Sigma_{c}$ (see (3.23) and (3.15)) made, essentially, of $P_{1}$ vectors (on a criss-cross grid) where the value at the "cross node" is adjusted in order to have a divergence which is constant in each square. This is what we call $P_{1}^{*}$. Hence we use here $\Sigma_{c} \subset H(\operatorname{div} ; \Omega)$ for approximating the vectors and $V_{c} \subset L^{2}(\Omega)$ for approximating the scalars (we always refer to the definitions (3.23) and (3.15)). From Theorem 3.2 and Lemma 3.9 we easily obtain (cf. also (3.33)) that this choice satisfies the inf-sup condition (3.2). Moreover, as $\operatorname{div}\left(\Sigma_{c}\right)=V_{c}$, the ellipticity in the kernel property (3.1) will also hold trivially.

\section{Numerical Results}

Let $\Omega$ be the square $] 0, \pi[\times] 0, \pi[$. Table 1 shows the first frequencies obtained using some of the mixed elements discussed in the previous section: Raviart-Thomas of lowest degree (RT), $P_{1}-\operatorname{div} P_{1}(\mathrm{P} 1)$ and $P_{1}^{*}-Q_{0}\left(\mathrm{P} 1^{*}\right)$. For all the elements, the $16 \times 16$ criss-cross mesh has been used.

We point out that only the RT element gives satisfactory results. In the other two elements spurious modes appear, which neither converge to any continuous eigenvalue nor tend to zero or to infinity.

We describe this behavior more precisely in Table 2 for the P1 element. We can observe that the fourth numerical eigenvalue seems to converge to 6 , which does not belong to the spectrum of the continuous problem. The P1 and P1* elements, even if they satisfy both conditions (3.1) and (3.2), give poor results for the approximation of problem (2.1). The presence of the spurious eigenvalues can be motivated by the fact that (3.1) and (3.2) are not sufficient conditions to ensure that the eigensolutions are "well approximated". In the next section we state the 
TABLE 1. Comparison of frequencies for different approximations

\begin{tabular}{ccccc}
\hline mode & exact & RT & P1 & P1* $^{*}$ \\
\hline$(1,1)$ & 2.00000 & 1.99786 & 2.00428 & 2.01286 \\
$(2,1)$ & 5.00000 & 4.99382 & 5.02674 & 5.08056 \\
$(1,2)$ & 5.00000 & 4.99382 & 5.02674 & 5.08056 \\
& & & 5.98074 & 6.03707 \\
$(2,2)$ & 8.00000 & 7.96568 & 8.06845 & 8.20593 \\
$(3,1)$ & 10.0000 & 9.99754 & 10.1067 & 10.3240 \\
$(1,3)$ & 10.0000 & 9.99754 & 10.1067 & 10.3240 \\
$(3,2)$ & 13.0000 & 12.9292 & 13.1804 & 13.5448 \\
$(2,3)$ & 13.0000 & 12.9292 & 13.1804 & 13.5448 \\
& & & 14.7166 & 15.0528 \\
& & & 14.7166 & 15.0528 \\
$(4,1)$ & 17.0000 & 17.0241 & 17.3073 & 17.9431 \\
$(1,4)$ & 17.0000 & 17.0241 & 17.3073 & 17.9431 \\
$(3,3)$ & 18.0000 & 17.8258 & 18.3456 & 19.0411 \\
$(4,2)$ & 20.0000 & 19.8995 & 20.4254 & 21.2951 \\
$(2,4)$ & 20.0000 & 19.8995 & 20.4254 & 21.2951 \\
\hline
\end{tabular}

TABLE 2. Nodal approximation on criss-cross mesh

\begin{tabular}{rrrrr}
\hline exact & \multicolumn{5}{c}{ computed } \\
\hline 2.00000 & 2.01711 & 2.00761 & 2.00428 & 2.00274 \\
5.00000 & 5.10637 & 5.04748 & 5.02674 & 5.01712 \\
5.00000 & 5.10637 & 5.04748 & 5.02674 & 5.01712 \\
& 5.92302 & 5.96578 & 5.98074 & 5.98767 \\
8.00000 & 8.27150 & 8.12152 & 8.06845 & 8.04383 \\
10.0000 & 10.4196 & 10.1890 & 10.1067 & 10.0684 \\
10.0000 & 10.4196 & 10.1890 & 10.1067 & 10.0684 \\
13.0000 & 13.7043 & 13.3195 & 13.1804 & 13.1156 \\
13.0000 & 13.7043 & 13.3195 & 13.1804 & 13.1156 \\
& 13.9669 & 14.5093 & 14.7166 & 14.8163 \\
& 13.9669 & 14.5093 & 14.7166 & 14.8163 \\
17.0000 & 18.1841 & 17.5423 & 17.3073 & 17.1972 \\
17.0000 & 18.1841 & 17.5423 & 17.3073 & 17.1972 \\
\hline mesh & $8 \times 8$ & $12 \times 12$ & $16 \times 16$ & $20 \times 20$ \\
\hline
\end{tabular}

meaning of "well-approximated eigensolutions" and we give a necessary condition for this property.

Remark 4.1. Although the $Q_{1}-P_{0}$ element does not satisfy the inf-sup condition, the eigenvalues computed by this method behave like those of the P1 and P1* 
methods. In this case the first spurious eigenvalue converges to 18 (actually, 18 is a simple eigenvalue of the Dirichlet problem in the square, while in the numerical computation it is approximated by two distinct modes).

For the Neumann problem the following explicit formula of the numerical eigenvalues computed by the $Q_{1}-P_{0}$ element is available (see [4]):

$$
\lambda_{h}^{m n}=\left(4 / h^{2}\right) \frac{\sin ^{2}\left(\frac{m h}{2}\right)+\sin ^{2}\left(\frac{n h}{2}\right)-2 \sin ^{2}\left(\frac{m h}{2}\right) \sin ^{2}\left(\frac{n h}{2}\right)}{1-(2 / 3)\left(\sin ^{2}\left(\frac{m h}{2}\right)+\sin ^{2}\left(\frac{n h}{2}\right)\right)+(4 / 9) \sin ^{2}\left(\frac{m h}{2}\right) \sin ^{2}\left(\frac{n h}{2}\right)},
$$

for $0 \leq m, n \leq N-1$, with $m+n \neq 0$ and $h=\pi / N$.

It is easy to verify that for $m, n$ fixed $\lim _{h \rightarrow 0} \lambda_{h}^{m n}=m^{2}+n^{2}=\lambda^{m n}$, and hence $\lambda_{h}^{33} \rightarrow 18$; on the other hand we also have by (4.1) $\lim _{h \rightarrow 0} \lambda_{h}^{N-1 N-1}=18$.

\section{On the CONVERGENCE OF EIGENVALUeS AND EIGENVECTORS}

In this section, using the notation introduced in Section 2, we show that property (2.12) is a sufficient condition for the uniform convergence (2.11).

Theorem 5.1. Condition (2.12) implies the uniform convergence (2.11).

Proof. Let $f \in L^{2}(\Omega)$ be such that $\|f\|_{0}=1$. Since the eigenfunctions $u_{i}$, for $i \in \mathbf{N}$, are an orthonormal basis in $L^{2}(\Omega)$, we have

$$
f=\sum_{i=1}^{\infty} \alpha_{i} u_{i}, \text { where } \alpha_{i}=\left(f, u_{i}\right)
$$

and

$$
\|f\|_{0}^{2}=\sum_{i=1}^{\infty} \alpha_{i}^{2}=1
$$

Let $\mathbb{P}_{h}$ be the $L^{2}(\Omega)$-projection operator defined in (2.14); then we can write

$$
\mathbb{P}_{h} f=\sum_{i=1}^{N(h)} \alpha_{i}^{h} u_{i}^{h}, \text { where } \alpha_{i}^{h}=\left(f, u_{i}^{h}\right),
$$

and

$$
\left\|\mathbb{P}_{h} f\right\|_{0}^{2}=\sum_{i=1}^{N(h)}\left(\alpha_{i}^{h}\right)^{2} \leq\|f\|_{0}^{2}=1
$$

Due to the definition of $T_{h}$, we have $T_{h} f=T_{h} \mathbb{P}_{h} f$, so that we obtain

$$
\begin{aligned}
T f-T_{h} f & =T f-T_{h} \mathbb{P}_{h} f \\
& =T\left(\sum_{i=1}^{\infty} \alpha_{i} u_{i}\right)-T_{h}\left(\sum_{i=1}^{N(h)} \alpha_{i}^{h} u_{i}^{h}\right) \\
& =\sum_{i=1}^{\infty} \alpha_{i} T u_{i}-\sum_{i=1}^{N(h)} \alpha_{i}^{h} T_{h} u_{i}^{h} \\
& =\sum_{i=1}^{\infty} \frac{1}{\lambda_{i}} \alpha_{i} u_{i}-\sum_{i=1}^{N(h)} \alpha_{i}^{h} \frac{1}{\lambda_{i}^{h}} u_{i}^{h} .
\end{aligned}
$$


For every $N \in \mathbf{N}$ we set $M=m(N)$ as defined in (2.7). Then we can write

$$
\begin{aligned}
T f-T_{h} f= & \sum_{i=1}^{M} \frac{1}{\lambda_{i}}\left(\alpha_{i} u_{i}-\alpha_{i}^{h} u_{i}^{h}\right)+\sum_{i=1}^{M}\left(\frac{1}{\lambda_{i}}-\frac{1}{\lambda_{i}^{h}}\right) \alpha_{i}^{h} u_{i}^{h} \\
& -\sum_{i=M+1}^{N(h)} \frac{1}{\lambda_{i}^{h}} \alpha_{i}^{h} u_{i}^{h}+\sum_{i=M+1}^{\infty} \frac{1}{\lambda_{i}} \alpha_{i} u_{i} .
\end{aligned}
$$

Now fix a positive $\varepsilon$. The last term is bounded in norm by $1 / \lambda_{M+1}$ and is therefore smaller than $\varepsilon$ for $M$ big enough. The third term has a norm smaller than or equal to $1 / \lambda_{M+1}^{h}$. For $M$ fixed and $h$ small enough, it will also be smaller than $\varepsilon$. The same is true for the first two terms: due to (2.12), for $M$ fixed each one of them will have norm smaller than $\varepsilon$ for $h$ small enough, and the proof is complete.

Let us conclude this section by showing that (2.11) is false for the third choice of spaces presented in the previous section.

Theorem 5.2. Let $\Sigma_{h}$ and $V_{h}$ be defined as in (3.13). Then the sequence $\left\{T_{h}\right\}$ introduced in (2.8) does not converge to $T$ in the norm of $\mathcal{L}\left(L^{2}(\Omega)\right)$.

Proof. In order to prove that (2.11) is false, we construct a sequence $\left\{v_{h}^{*}\right\} \subset V_{h}$ such that

$$
\begin{array}{ll}
\left\|v_{h}^{*}\right\|_{0}=1 & \forall h>0, \\
\left\|T v_{h}^{*}-T_{h} v_{h}^{*}\right\|_{0} \nrightarrow 0 & \text { as } h \rightarrow 0 .
\end{array}
$$

We take $v_{h}^{*}=\tilde{v}_{h} /\left\|\tilde{v}_{h}\right\|_{0}$, where $\tilde{v}_{h}$ is defined in Remark 3.11. Hence (3.35) reduces to

$$
\left|\left(\operatorname{div} \underline{\tau}_{h}, v_{h}^{*}\right)\right| \leq C|| \underline{\tau}_{h} \|_{0} \quad \forall \underline{\tau}_{h} \in \Sigma_{h} .
$$

Since $\left\|v_{h}^{*}\right\|_{0}=1$ and $v_{h}^{*}$ has zero mean-value in each macroelement, the sequence $\left\{v_{h}^{*}\right\}$ converges weakly to zero in $L^{2}(\Omega)$. Owing to the compactness of $T$, it follows that

$$
T v_{h}^{*} \rightarrow 0 \quad \text { strongly in } L^{2}(\Omega) .
$$

Consider the solution $\left(\underline{\sigma}_{h}, u_{h}\right)$ of the problem

$$
\begin{cases}\left(\underline{\sigma}_{h}, \underline{\tau}_{h}\right)+\left(\operatorname{div} \underline{\tau}_{h}, u_{h}\right)=0 & \forall \underline{\tau}_{h} \in \Sigma_{h}, \\ \left(\operatorname{div} \underline{\sigma}_{h}, v_{h}\right)=-\left(v_{h}^{*}, v_{h}\right) & \forall v_{h} \in V_{h}\end{cases}
$$

We observe that by definition $u_{h}=T_{h} v_{h}^{*}$. Our aim is to prove that $\left\|u_{h}\right\|_{0} \not \rightarrow 0$.

From the second equation of (5.10) and the first of (5.7) we obtain

$$
\left|\left(\operatorname{div} \underline{\sigma}_{h}, u_{h}\right)\right|=\left|\left(v_{h}^{*}, u_{h}\right)\right| \leq\left\|u_{h}\right\|_{0},
$$

and from the first equation of (5.10)

$$
\left|\left(\operatorname{div} \underline{\sigma}_{h}, u_{h}\right)\right|=\left\|\underline{\sigma}_{h}\right\|_{0}^{2} .
$$

Using (5.8) and then the second equation of (5.10), we get

$$
\left\|\underline{\sigma}_{h}\right\|_{0} \geq \frac{1}{C}\left|\left(\operatorname{div} \underline{\sigma}_{h}, v_{h}^{*}\right)\right|=\frac{1}{C}\left(v_{h}^{*}, v_{h}^{*}\right)=\frac{1}{C} .
$$

Finally, putting together (5.11), (5.12) and (5.13), we obtain

$$
\left\|u_{h}\right\|_{0} \geq \frac{1}{C^{2}}
$$


This concludes the proof of $(5.7)$, because $T v_{h}^{*}$ tends to zero as $h \rightarrow 0$ while $u_{h}=T_{h} v_{h}^{*}$ does not.

Remark 5.3. A similar proof shows that (2.11) does not hold for the $Q_{1}-P_{0}$ element of subsection 3.2, nor for the $P_{1}^{*}-Q_{0}$ element of subsection 3.4. On the other hand, from (3.5) it follows that (2.11) holds for the mixed approach of subsection 3.1.

\section{ERror estimates}

The aim of this section is to recall, for convenience of the reader, the proof of the good behavior of the mixed approach described in subsection 3.1 when applied to problem (2.1). In particular we shall prove the uniform convergence (2.11), together with the error estimates for eigenvalues and eigenvectors for a general choice of spaces $\Sigma_{h}$ and $V_{h}$ satisfying some suitable abstract conditions. Results of this type are well known. For instance, the specific case of Raviart-Thomas elements can be found in [1], together with an abstract framework and several references.

We introduce the operator $S: L^{2}(\Omega) \rightarrow H(\operatorname{div} ; \Omega)$ given by

$$
\begin{cases}(S f, \underline{\tau})+(\operatorname{div} \underline{\tau}, u)=0 & \forall \underline{\tau} \in H(\operatorname{div} ; \Omega) \\ (\operatorname{div} S f, v)=-(f, v) & \forall v \in L^{2}(\Omega)\end{cases}
$$

and $\Sigma_{0}=S\left(L^{2}(\Omega)\right)$, which due to the regularity assumption on $\Omega$ satisfies

$$
\Sigma_{0} \subset H^{1}(\Omega)^{2} .
$$

Let us recall the so-called Fortin's operator (see [10]) $\Pi_{h}: \Sigma_{0} \rightarrow \Sigma_{h}$ :

$$
\begin{aligned}
& \left(\operatorname{div}\left(\underline{\sigma}-\Pi_{h} \underline{\sigma}\right), v_{h}\right)=0 \quad \forall v_{h} \in V_{h}, \\
& \left\|\Pi_{h} \underline{\sigma}\right\|_{\operatorname{div}} \leq C\|\underline{\sigma}\|_{1} .
\end{aligned}
$$

Proposition 6.1. Let $f \in L^{2}(\Omega)$ be given. Suppose the existence of $\Pi_{h}: \Sigma_{0} \rightarrow \Sigma_{h}$ satisfying (6.3). Assume moreover the ellipticity in the kernel property (3.1). Then, using the notation of (2.3) and (2.8), the following estimates hold:

$$
\begin{aligned}
& \left\|\underline{\sigma}-\underline{\sigma}_{h}\right\|_{0} \leq C\left(\left\|\underline{\sigma}-\Pi_{h} \underline{\sigma}\right\|_{0}+\frac{1}{\sqrt{\alpha}} \inf _{v_{h} \in V_{h}}\left\|T f-v_{h}\right\|_{0}\right), \\
& \left\|T f-T_{h} f\right\|_{0} \leq C\left(\inf _{v_{h} \in V_{h}}\left\|T f-v_{h}\right\|_{0}+\left\|\underline{\sigma}-\underline{\sigma}_{h}\right\|_{0}\right) .
\end{aligned}
$$

Proof. The result is essentially known (see e.g. [9, 12, 1, 8] for results of this type). However, for convenience of the reader, we give the idea of the proof. In order to estimate the difference $\left\|\mathbb{P}_{h} T f-T_{h} f\right\|_{0}$ we can use the inf-sup condition which is implied by the existence of $\Pi_{h}$ :

$$
\begin{aligned}
& \left\|\mathbb{P}_{h} T f-T_{h} f\right\|_{0} \leq C \sup _{\underline{\tau} \in \Sigma_{h}} \frac{\left(\mathbb{P} h T f-T_{h} f, \operatorname{div} \underline{\tau}\right)}{\|\underline{\tau}\|_{\operatorname{div}}} \\
& \leq C \sup _{\underline{\tau} \in \Sigma_{h}} \frac{(\mathbb{P} h T f-T f, \operatorname{div} \underline{\tau})+\left(T f-T_{h} f, \operatorname{div} \underline{\tau}\right)}{\|\underline{\tau}\|_{\operatorname{div}}} \\
& \leq C\left\|I P_{h} T f-T f\right\|_{0}+\sup _{\underline{\tau} \in \Sigma_{h}} \frac{-\left(\underline{\sigma}-\underline{\sigma}_{h}, \underline{\tau}\right)}{\|\underline{\tau}\|_{\text {div }}} \\
& \leq C\left\|\mathbb{P}_{h} T f-T f\right\|_{0}+\left\|\underline{\sigma}-\underline{\sigma}_{h}\right\|_{0} .
\end{aligned}
$$

The second estimate of (6.4) is then obtained by the triangle inequality. Finally, the first one can be easily deduced using again the triangle inequality, the error 
equations and the ellipticity in the kernel property (3.1):

$$
\begin{aligned}
& \left\|\Pi_{h} \underline{\sigma}-\underline{\sigma}_{h}\right\|_{0}^{2}=\left(\Pi_{h} \underline{\sigma}-\underline{\sigma}, \Pi_{h} \underline{\sigma}-\underline{\sigma}_{h}\right)+\left(\underline{\sigma}-\underline{\sigma}_{h}, \Pi_{h} \underline{\sigma}-\underline{\sigma}_{h}\right) \\
& \quad=\left(\Pi_{h} \underline{\sigma}-\underline{\sigma}, \Pi_{h} \underline{\sigma}-\underline{\sigma}_{h}\right)-\left(\operatorname{div}\left(\Pi_{h} \underline{\sigma}-\underline{\sigma}_{h}\right), T f-\mathbb{P}_{h} T f\right) \\
& \quad \leq\left\|\Pi_{h} \underline{\sigma}-\underline{\sigma}_{h}\right\|_{0}\left\|\underline{\sigma}-\Pi_{h} \underline{\sigma}\right\|_{0}+\left\|\operatorname{div}\left(\Pi_{h} \underline{\sigma}-\underline{\sigma}_{h}\right)\right\|_{0}\|T f-\mathbb{P} h T f\|_{0} \\
& \quad \leq\left\|\Pi_{h} \underline{\sigma}-\underline{\sigma}_{h}\right\|_{0}\left(\left\|\underline{\sigma}-\Pi_{h} \underline{\sigma}\right\|_{0}+\frac{1}{\sqrt{\alpha}}\left\|T f-\mathbb{P}_{h} T f\right\|_{0}\right) .
\end{aligned}
$$

Remark 6.1. It is well known that the existence of the operator $\Pi_{h}$ verifying (6.3) together with (3.1) implies the following error estimate for problem (2.8):

$$
\left\|\underline{\sigma}-\underline{\sigma}_{h}\right\|_{\text {div }} \leq C\left(\inf _{v_{h} \in V_{h}}\left\|u-v_{h}\right\|_{0}+\inf _{\underline{x}_{h} \in \Sigma_{h}}\left\|\underline{\sigma}-\underline{\tau}_{h}\right\|_{\text {div }}\right) .
$$

Actually, estimates (6.4) and (6.7) are not enough to ensure the uniform convergence (2.11). This has been proved with the counterexample given in the previous section. The $P_{1}-\operatorname{div} P_{1}$ element and the $P_{1}^{*}-Q_{0}$ element on a criss-cross mesh satisfy both (3.1) and (3.2). Moreover, it is not difficult to show the existence of an operator $\Pi_{h}$ which satisfies Fortin's hypothesis (6.3). However, they do not satisfy the uniform convergence (2.11); hence they are not well suited for the approximation of eigenproblem (2.1), as has been proved in the previous section and numerically demonstrated in Section 4.

From Proposition 6.1 it follows that it will be sufficient to add the following hypothesis for the uniform convergence (2.11):

$$
\left\|I-\Pi_{h}\right\|_{\mathcal{L}\left(\Sigma_{0}, L^{2}(\Omega)^{2}\right)} \rightarrow 0 .
$$

The following theorem gives the error estimates for eigenproblem (2.2).

Theorem 6.2. Assume that there exists a linear operator $\Pi_{h}: \Sigma_{0} \rightarrow \Sigma_{h}$ which satisfies Fortin's conditions (6.3) and (6.8).

Assume also the ellipticity in the kernel property (3.1).

For every $N \in \mathbf{N}$ define moreover the following function $\rho_{N}:[0,1] \rightarrow \mathbf{R}$ :

$$
\rho_{N}(h)=\sup _{u \in \oplus_{i=1}^{m(N)} E_{i}}\left(\inf _{v_{h} \in V_{h}}\left\|u-v_{h}\right\|_{0}+\left\|\underline{\nabla} u-\Pi_{h} \underline{\nabla} u\right\|_{0}\right) .
$$

Then problem (2.2) is well posed and the following error estimates hold true with $C$ independent of $h$ :

$$
\begin{aligned}
& \sum_{i=1}^{m(N)}\left|\lambda_{i}-\lambda_{i}^{h}\right| \leq C\left(\rho_{N}(h)\right)^{2} \\
& \hat{\delta}\left(\bigoplus_{i=1}^{m(N)} E_{i}, \bigoplus_{i=1}^{m(N)} E_{i}^{h}\right) \leq C \rho_{N}(h)
\end{aligned}
$$


Proof. The proof is an immediate consequence of estimate (6.4) of Proposition 6.1, the definition (6.9) of $\rho_{N}$ and classical results on eigenvalues approximation (see [15], (3.17), (3.18) for the derivation of estimates (6.10); see also the references therein).

Remark 6.3. This last theorem implies, in particular, that the mixed spaces recalled in Section 3 give good results for the approximation of problem (2.1). For instance, when using the RT elements of lowest degree it is well known that for $N$ fixed one has $\rho_{N}(h)=O(h)$ (this is also easy to check using (3.4) and (6.9)).

\section{REFERENCES}

1. I. Babuška and John E. Osborn, Eigenvalue problems, Handbook of Numerical Analysis (P.G. Ciarlet and J.L. Lions, eds.), vol. II, Elsevier Science Publishers B.V., North Holland, 1991, pp. 641-788. MR 92f:65001

2. K.-J. Bathe, C. Nitikitpaiboon, and X. Wang, A mixed displacement-based finite element formulation for acoustic fluid-structure interaction, Computers \& Structures 56 (1995), 225237. MR 96b:73094

3. A. Bermúdez and D.G. Pedreira, Mathematical analysis of a finite element method without spurious solutions for computation od dieletric waveguides, Numer. Math. 61 (1992), 39-57. MR 92m:65139

4. D. Boffi, R. Duran, and L. Gastaldi, A remark on spurious eigenvalues in a square, Appl. Math. Lett. (1998), to appear.

5. D. Boffi, P. Fernandes, L. Gastaldi, and I. Perugia, Edge approximation of eigenvalue problems arising from electromagnetics, Numerical Methods in Engineering '96 (Désidéri, Le Tallec, Oñate, Périaux, and Stein, eds.), John Wiley \& Sons, 1996, pp. 551-556.

6. __ Computational models of electromagnetic resonators: analysis of edge element approximation, SIAM J. Numer. Anal. (1998), To appear.

7. J.M. Boland and R. Nicolaides, Stable and semistable low order finite elements for viscous flows, SIAM J. Numer. Anal. 22 (1985), 474-492. MR 86m:65139

8. F. Brezzi and M. Fortin, Mixed and hybrid finite element methods, Springer-Verlag, New York, 1991. MR 92d:65187

9. J. Falk and John E. Osborn, Error estimates for mixed methods, R.A.I.R.O. Anal. Numer. 4 (1980), 249-277. MR 82j:65076

10. M. Fortin, An analysis of the convergence of mixed finite element methods, R.A.I.R.O. Anal. Numer. 11 (1977), 341-354. MR 55:4473

11. L. Gastaldi, Mixed finite element methods in fluid structure systems, Numer. Math. 74 (1996), 153-176. MR 97e:73053

12. V. Girault and P.A. Raviart, Finite element methods for Navier-Stokes equations, theory and algorithms, Springer-Verlag, Berlin, 1986. MR 88b:65129

13. C. Johnson and J. Pitkäranta, Analysis of some mixed finite element methods related to reduced integration, Math. Comp. 38 (1982), 375-400. MR 83d:65287

14. W.G. Kolata, Approximation in variationally posed eigenvalue problems, Numer. Math. 29 (1978), 159-171. MR 80a:49077

15. B. Mercier, J. Osborn, J. Rappaz, and P.A. Raviart, Eigenvalue approximation by mixed and hybrid methods, Math. Comp. 36 (1981), 427-453. MR 82b:65108

16. John E. Osborn, Eigenvalue approximations by mixed methods, Advances in Computer Methods for Partial Differential Equations III (R. Vichnevetsky and R. Stepleman, eds.), New Brunswick, 1979, pp. 158-161. MR 82b:65024

17. J. Qin, On the convergence of some low order mixed finite elements for incompressible fluids, Ph.D. thesis, The Pennsylvania State University, Department of Mathematics, 1994.

18. X. Wang and K.-J. Bathe, On mixed elements for acoustic fluid-structure interactions, Math. Models Methods Appl. Sci. 7 (1997), no. 3, 329-343. MR 98c:73068

19. J.P. Webb, Edge elements and what they can do for you, IEEE Trans. on Magnetics 29 (1993), 1460-1465. 
Dipartimento di Matematica "F. Casorati", Università di Pavia, 27100 Pavia, Italy

E-mail address: boffi@ian.pv.cnr.it

Dipartimento di Matematica "F. Casorati", Università di Pavia and Istituto di Analisi Numerica DEL C.N.R., 27100 PAVIA, Italy

E-mail address: brezzi@ian.pv.cnr.it

Dipartimento di Matematica, Università di Roma "La Sapienza", 00185 Roma, italy

E-mail address: gastaldi@ian.pv.cnr.it 